\title{
Beneficial effect of anti-diabetic drugs for nonalcoholic fatty liver disease
}

\author{
Kyung-Soo Kim ${ }^{1}$ and Byung-Wan Lee ${ }^{2}$ \\ 'Division of Endocrinology and Metabolism, Department of Internal Medicine, CHA Bundang Medical Center, CHA University School of \\ Medicine, Seongnam; 'Division of Endocrinology and Metabolism, Department of Internal Medicine, Yonsei University College of \\ Medicine, Seoul, Korea
}

Nonalcoholic fatty liver disease (NAFLD) is the most common liver disorder and is associated with various metabolic diseases, including type 2 diabetes mellitus. There are no approved drugs for NAFLD, and the only approved treatment option is weight reduction. As insulin resistance plays an important role in the development of NAFLD, many antidiabetic drugs have been evaluated for the treatment of NAFLD. Improvement of liver enzymes has been demonstrated by many anti-diabetic drugs, but histological assessment still remains insufficient. Pioglitazone could become the firstline therapy for T2DM patients with NAFLD, based on evidence of histological improvement in patients with biopsyproven nonalcoholic steatohepatitis (NASH). Liraglutide, another promising alternative, is not yet recommended in patients with NAFLD/NASH due to limited evidence. Therefore, well-designed randomized controlled trials should be performed in the near future to demonstrate if and how anti-diabetic drugs can play a role in the treatment of NAFLD. (Clin Mol Hepatol 2020;26:430-443)

Keywords: Anti-diabetic drug; Diabetes mellitus; Metabolic diseases; Non-alcoholic fatty liver disease; Treatment

\section{INTRODUCTION}

Nonalcoholic fatty liver disease (NAFLD) is associated with liverrelated morbidity, including progression to nonalcoholic steato- hepatitis (NASH), advanced fibrosis, and hepatocellular carcinoma (HCC). NAFLD is the fastest growing cause of HCC in liver transplant candidates, ${ }^{1}$ and it is projected that NAFLD is becoming the leading cause of liver transplantation. ${ }^{2}$ NAFLD can be coexistent

\footnotetext{
Abbreviations:

ALT, alanine aminotransferase; AMPK, adenosine monophosphate-activated protein kinase; AST, aspartate aminotransferase; AWARD, Assessment of Weekly Administration of LY2189265 (dulaglutide) in Diabetes; BMI, body mass index; CV, cardiovascular; DEAN, Dapagliflozin Efficacy and Action in NASH; DPP4, dipeptidyl peptidase-4; E-LIFT, Effect of Empagliflozin on Liver Fat Content in Patients With Type 2 Diabetes; EFFECT-II, Effects of Omega-3 Carboxylic Acids and Dapagliflozin on Liver Fat Content in Diabetic Patients; EMPAREG OUTCOME, Empagliflozin Cardiovascular Outcome Event Trial in Type 2 Diabetes Mellitus Patients; GIP, glucose-dependent insulinotropic polypeptide; GLP-1 RAs, GLP-1 receptor agonists; GLP-1, glucagon-like peptide-1; HCC, hepatocellular carcinoma; LEAD, Liraglutide Effect and Action in Diabetes; MRI, magnetic resonance imaging; MRS, magnetic resonance spectroscopy; NAFLD, nonalcoholic fatty liver disease; NAS, NAFLD activity score; NASH, nonalcoholic steatohepatitis; PDFF, proton density fat fraction; PIVENS, Pioglitazone versus Vitamin E versus Placebo for the Treatment of Nondiabetic Patients with NASH; PPAR- $\gamma$, peroxisome proliferator-activated receptor- $-;$ RCTs, randomized controlled trials; SGLT2, sodium-glucose cotransporter 2; T2DM, type 2 diabetes mellitus; TNF, tumor necrosis factor; TZDs, thiazolidinediones
}

\section{Corresponding author: Byung-Wan Lee}

Division of Endocrinology and Metabolism, Department of Internal Medicine, Yonsei University College of Medicine, 50-1 Yonsei-ro, Seodaemun-gu, Seoul 03722, Korea

Tel: +82-2-2228-1938, Fax: +82-2-393-6884

E-mail: bwanlee@yuhs.ac

https://orcid.org/0000-0002-9899-4992 
with type 2 diabetes mellitus (T2DM), and this combination results in the undesirable consequence of cardiovascular (CV) events, which underscores the need for lifestyle modification in the management of NAFLD regardless of diabetes. ${ }^{3,4}$ NAFLD is the one of the most common liver disorders, affecting approximately $20-40 \%$ of the population worldwide, with different burdens according to age, sex, ethnicity, and diagnostic tools. ${ }^{5,6}$ The presence of diabetes is closely associated with the severity of NAFLD, progression to NASH, advanced fibrosis, cirrhosis, and even $\mathrm{HCC}_{1}^{7-9}$ independently of liver enzymes. ${ }^{10,11}$ Studies on South Korean patients with T2DM who underwent ultrasonography in a university and a private clinic-based diabetes care facility showed $63.3 \%$ and $72.7 \%$ prevalence of NAFLD, respectively. ${ }^{12,13}$ A recent metaanalysis reported that the global prevalence of NAFLD and NASH among patients with $\mathrm{T} 2 \mathrm{DM}$ is $55.5 \%$ and $37.3 \%$, respectively. ${ }^{14}$ The European Association for the Study of the Liver, the European



Figure 1. Mechanism of action and effects of anti-diabetic drugs on glucose metabolism. DPP-4, dipeptidyl peptidase-4; GLP-1 RA, glucagon-like peptide-1 receptor agonist; SGLT2, sodium-glucose cotransporter 2. 
Association for the Study of Diabetes, and the European Association for the Study of Obesity, in their joint clinical practice guidelines for the management of NAFLD recommend screening for NAFLD in T2DM patients and vice versa. ${ }^{15}$ Although weight reduction is the cornerstone of therapy among the various treatments for NAFLD, ${ }^{16}$ pharmacotherapy should be administered since sufficient weight reduction can be difficult to achieve and maintain. In subjects with T2DM and NAFLD, management with anti-diabetic drugs is more effective than lifestyle modification in controlling glucose level (Fig. 1). Moreover, lifestyle changes, together with anti-diabetic medications, are likely to have an additive effect on reducing the risk factors associated with CV diseases, decreasing hepatic fat accumulation, and delaying progression to NASH and fibrosis. Although there is no approved drug of choice for managing NAFLD, many anti-diabetic drugs have been tested in patients with NAFLD due to the shared epidemiological and pathophysiological features between NAFLD and T2DM. The aim of this review is to summarize the beneficial effects of anti-diabetic drugs for NAFLD.

\section{METFORMIN}

Metformin has been derived from the guanidine-rich Galega officinalis (goat's rue or French lilac), and is a kind of general insulin sensitizer. ${ }^{17}$ Although its molecular mechanisms of action are complex and not completely understood, metformin has been shown to act via both adenosine monophosphate-activated protein kinase (AMPK)-dependent and AMPK-independent mechanisms, by the inhibition of mitochondrial respiration but also perhaps by the inhibition of mitochondrial glycerophosphate dehydrogenase and a mechanism involving lysosomes at the molecular level (Table 1). ${ }^{18}$ Physiologically, metformin reduces blood glucose level by dominantly inhibiting hepatic glucose production via AMPK activation, stimulation of muscle glucose uptake, and decrease of intestinal glucose absorption. ${ }^{18}$ All current guidelines recommend metformin as the first-line agent for T2DM. ${ }^{19,20}$

Since metformin improves insulin resistance, which is the major pathogenesis of NAFLD, it has been widely tested in both animal NAFLD models and human clinical trials. In the insulin-resistant ob/ob mouse model with hepatic steatosis, metformin improved fatty liver disease and reversed the hepatomegaly, steatosis, and alanine aminotransferase (ALT) level by decreasing the expression of tumor necrosis factor-alpha (TNF-a) and TNF-inducible factors that promote hepatic lipid accumulation and adenosine triphos- phate depletion ${ }^{21}$ or autophagy activation. ${ }^{22}$ In a pilot study of 20 non-diabetic patients with NAFLD, metformin treatment for 4 months reduced liver enzymes and improved insulin sensitivity and liver volume as detected by ultrasound. ${ }^{23}$ However, among well-designed randomized controlled trials (RCTs), the TONIC trial, which recruited 173 non-diabetic patients aged 8-17 years with biopsy-proven NAFLD and persistent increase in ALT, did not demonstrate reduced ALT level and improved liver histology with metformin compared to placebo. ${ }^{24}$ To date, meta-analyses have shown that metformin could improve liver function, insulin resistance, and body mass index (BMI) to some extent, but does not impact histological response in NAFLD patients. ${ }^{25-27}$ In this regard, current guidelines do not recommend metformin for treating NASH in adult patients. ${ }^{15,28}$ Despite the lack of known benefits to treat NAFLD, metformin aids in reducing CV risks related to diabetes. Furthermore, a meta-analysis of case-control studies found that metformin use was associated with a reduced risk of HCC, which is the final sequence of NAFLD, and it may be a relevant factor in preventing HCC in diabetic patients. ${ }^{29}$ Based on these studies, metformin could be beneficial for NAFLD patients with T2DM.

\section{THIAZOLIDINEDIONES}

Thiazolidinediones (TZDS) are peroxisome proliferator-activated receptor- $\gamma$ (PPAR- $-\gamma$ ) agonists that act as insulin sensitizers on adipose tissue, muscle, and liver. TZDs improve insulin and glucose parameters, modulate adipose tissue distribution while decreasing visceral fat and increasing subcutaneous fat, and reduce lipotoxicity in the liver. In addition, TZDs have demonstrated anti-inflammatory and antioxidant properties.

\section{Pioglitazone}

Pioglitazone was widely evaluated for the treatment of NAFLD. Many RCTs have shown histological improvement in steatosis and lobular inflammation after pioglitazone treatment. In a pilot study, pioglitazone treatment for 48 weeks showed histological improvement (steatosis, parenchymal inflammation, cellular injury, and Mallory bodies) in 18 non-diabetic patients with biopsy-proven NASH ${ }^{30}$ In 55 patients with impaired glucose tolerance or T2DM (mean HbA1c 6.2\%), pioglitazone treatment ( $45 \mathrm{mg}$ daily) with a hypocaloric diet for 6 months resulted in improvements in insulin sensitivity, ALT levels, steatosis, ballooning necrosis, and inflam- 
Table 1. Molecular mechanisms of anti-diabetic drugs and their effects on nonalcoholic fatty liver disease

\begin{tabular}{|c|c|c|c|}
\hline Anti-diabetic drugs & Molecular mechanisms & Weight change & Effects on NAFLD \\
\hline Metformin & $\begin{array}{l}\text { AMPK-dependent mechanisms } \\
\text { - Inhibits the mitochondrial respiratory chain in the liver, leading } \\
\text { to activation of AMPK, enhancing insulin sensitivity (via effects } \\
\text { on fat metabolism), and lowering CAMP, and thereby reducing } \\
\text { the expression of gluconeogenic enzymes } \\
\text { AMPK-independent mechanisms } \\
\text { - Inhibits fructose-1,6-bisphosphatase by AMP }\end{array}$ & $\begin{array}{l}\text { Neutral (potential } \\
\text { modest loss) }\end{array}$ & $\begin{array}{l}\text { - Improvement of liver enzymes } \\
\text { and liver fat content }\end{array}$ \\
\hline Thiazolidinediones & $\begin{array}{l}\text { Activation of the transcription factor PPAR- } \gamma \text { involved in the } \\
\text { transcription of genes regulating glucose and fat metabolism } \\
\rightarrow \text { When activated by a ligand, PPAR- } \gamma \text { binds to the retinoid X } \\
\text { receptor (RXR) to form a heterodimer. } \\
\rightarrow \text { This binds to DNA to regulate the genetic transcription } \\
\text { and translation of various proteins involved in cellular } \\
\text { differentiation as well as glucose and lipid metabolism. }\end{array}$ & Gain & $\begin{array}{l}\text { Pioglitazone } \\
\text { - Improvement of liver enzymes } \\
\text { and liver fat content } \\
\text { - Some improvement on } \\
\text { histological features of NASH } \\
\text { Rosiglitazone and lobeglitazone } \\
\text { - Improvement of liver enzymes } \\
\text { and liver fat content }\end{array}$ \\
\hline GLP1-RAs & $\begin{array}{l}\text { Binding of GLP-1 receptor (pancreatic } \beta \text {-cells) } \\
\rightarrow \text { Elicits its effects via acute elevation in CAMP levels } \\
\rightarrow \text { Subsequent protein kinase(s) activation } \\
\rightarrow \text { Elevated } \mathrm{Ca}^{2+} \text { and ATP } \\
\rightarrow \text { Stimulates insulin secretion }\end{array}$ & Loss & $\begin{array}{l}\text { Liraglutide } \\
\text { - Improvement of liver enzymes } \\
\text { and liver fat content } \\
\text { - Potential beneficial effect on } \\
\text { histological features of NASH } \\
\text { Exenatide and dulaglutide } \\
\text { - Improvement of liver enzymes } \\
\text { and liver fat content } \\
\text { Lixisenatide and semaglutide } \\
\text { - Improvement of liver enzymes }\end{array}$ \\
\hline DPP-4 inhibitors & $\begin{array}{l}\text { Competitively inhibits the enzyme DPP-4; this enzyme breaks } \\
\text { down the incretin GLP-1 and GIP, which are released in } \\
\text { response to a meal } \\
\text { (DPP-4 is a } 766 \text { amino acid amino peptidase, and is a tetramer } \\
\text { with each subunit consisting of two domains: N-terminal } \\
\text { B-propeller domain \& C-terminal catalytic domain). }\end{array}$ & Neutral & $\begin{array}{l}\text { Sitagliptin and vildagliptin } \\
\text { - Improvement of liver enzymes } \\
\text { and liver fat content }\end{array}$ \\
\hline SGLT2 inhibitors & $\begin{array}{l}\text { Inhibits SGLT2 action and causes reduction in renal glucose } \\
\text { reabsorption } \\
\text { (SGLT2 is expressed in the luminal membrane of the proximal } \\
\text { tubule [mainly } 51 \text { and S2 segment]. It reabsorbs } \sim 97 \% \text { of } \\
\text { filtered glucose under normoglycemic conditions). }\end{array}$ & Loss & $\begin{array}{l}\text { Dapagliflozin, canagliflozin, } \\
\text { empagliflozin, ipragliflozin, and } \\
\text { luseogliflozin } \\
\text { - Improvement of liver enzymes } \\
\text { and liver fat content }\end{array}$ \\
\hline $\begin{array}{l}\text { a-glucosidase } \\
\text { inhibitors }\end{array}$ & $\begin{array}{l}\text { Inhibits intestinal a-glucosidase } \\
\text { (Enzyme a-glucosidase is located in the brush border of the } \\
\text { enterocytes of the jejunum in the small intestine, and happens } \\
\text { to be a key enzyme in carbohydrate synthesis and breakdown. } \\
\text { It is an exo-type carbohydrolase, which cleaves glycosidic } \\
\text { bonds in complex carbohydrate to release absorbable } \\
\text { monosaccharides). }\end{array}$ & Neutral & No study in human \\
\hline Sulfonylureas & $\begin{array}{l}\text { Binds to sulfonylurea receptors and causes closing of } \mathrm{K}_{\text {ATP }} \\
\text { channels on } \beta \text {-cell plasma membranes, influx of } \mathrm{Ca}^{2+} \text {, and } \\
\text { stimulation of insulin release }\end{array}$ & Gain & No study in human \\
\hline Meglitinides & $\begin{array}{l}\text { Binds to sulfonylurea receptors, but at different binding sites to } \\
\text { sulfonylurea causes closing of } \mathrm{K}_{\text {ATp }} \text { channels on b-cell plasma } \\
\text { membranes, influx of } \mathrm{Ca}^{2+} \text {, and stimulation of insulin release }\end{array}$ & Gain & No study in human \\
\hline
\end{tabular}

NAFLD, nonalcoholic fatty liver disease; AMPK, adenosine monophosphate-activated protein kinase; CAMP, cyclic adenosine monophosphate; PPAR- $\gamma$, peroxisome proliferator-activated receptor- $\gamma ;$ NASH, nonalcoholic steatohepatitis; ATP, adenosine triphosphate; GLP1-RAs, GLP-1 receptor agonists; DPP-4, dipeptidyl peptidase-4; GLP-1, glucagon-like peptide-1; GIP, glucose-dependent insulinotropic polypeptide; SGLT2, sodium-glucose cotransporter 2. 
mation of liver biopsy-confirmed NASH patients. ${ }^{31}$ Meta-analyses agreed with the beneficial effects of pioglitazone on lobular inflammation. ${ }^{26,32,33}$ However, there were conflicting results for hepatic fibrosis. The landmark phase 3 PIVENS (Pioglitazone versus Vitamin E versus Placebo for the Treatment of Nondiabetic Patients with NASH) trial was conducted as a multicenter, randomized, placebo-controlled, double-blind clinical trial of pioglitazone compared to vitamin $\mathrm{E}$ in the treatment of NASH, by enrolling 247 adults without diabetes who had biopsy-confirmed NASH. ${ }^{34}$ After a 96-week treatment period, $45 \mathrm{mg} /$ day pioglitazone treatment was associated with reductions in serum ALT levels $(P<0.001)$, hepatic steatosis $(P<0.001)$, and lobular inflammation $(P=0.004)$, but there was no improvement in fibrosis scores. On the contrary, another RCT was conducted in 101 patients with biopsy-proven $\mathrm{NASH}$ to investigate whether $45 \mathrm{mg} /$ day pioglitazone treatment for 18 months could achieve the primary outcome with an improvement of at least two points in NAFLD activity score (NAS) and no worsening of fibrosis. ${ }^{35}$ Pioglitazone treatment showed $58 \%$ achievement in the primary outcome and $51 \%$ resolution of NASH ( $P<0.001$ for each). Pioglitazone treatment also was associated with improvement in individual histologic scores, including fibrosis score $(P=0.039)$; reduced hepatic triglyceride content from $19 \%$ to $7 \%(P<0.001)$; and improved adipose tissue, hepatic, and muscle insulin sensitivity $(P<0.001$ vs. placebo for all). In advanced fibrosis (stages F3-F4), meta-analysis showed that pioglitazone use improved fibrosis in patients with NASH and T2DM, as well as in patients without diabetes. ${ }^{36}$ Therefore, pioglitazone could be a first-line therapy for patients with NASH and T2DM. However, due to limited evidence, pioglitazone should be cautiously used in subjects without T2DM to treat NAFLD without biopsy-proven NASH. ${ }^{28}$ It is important to remember that weight gain, fluid retention, and bone loss are common side effects of pioglitazone treatment. In this regard, the risks and benefits should be considered before starting therapy.

\section{Rosiglitazone}

Rosiglitazone was once a popular TZD, but it was withdrawn from some markets in 2010 due to a high risk of myocardial infarction. In many RCTs, rosiglitazone demonstrated a biochemical improvement in liver enzymes and glycemic control in both diabetic and nondiabetic patients with NAFLD, whereas evidence of histological improvement was less clear. ${ }^{37}$ Although no firm association was found after an extensive review of all evidence by the United States Food and Drug Administration, rosiglitazone is no longer available in most countries, and its prescribing remains severely restricted in the United States.

\section{Lobeglitazone}

Lobeglitazone is a novel PPAR- $\gamma$ agonist developed in South Korea, and it has been used for patients with T2DM in several countries. It exhibited similar beneficial effects to other TZDs, such as glucose-lowering, anti-inflammatory, and antioxidant actions. ${ }^{38}$ Regarding NAFLD, lobeglitazone treatment has limited evidence. In high-fat diet-induced obese mice, lobeglitazone decreased hepatic steatosis, attenuated lipogenesis-induced oxidative liver injury, and reversed hepatic lipid dysregulation. ${ }^{39,40}$ In a multicenter, prospective, open-label, exploratory clinical trial, 43 patients with NAFLD and T2DM who were treated daily with $0.5 \mathrm{mg}$ lobeglitazone for 24 weeks experienced improved liver enzymes and ameliorated hepatic fat content, as assessed by vibration-controlled transient elastography, with controlled attenuation parameters. ${ }^{41}$ Recommendations for the use of lobeglitazone are similar to those of pioglitazone.

\section{GLUCAGON-LIKE PEPTIDE-1 (GLP-1) RECEPTOR AGONISTS}

GLP-1 is released from intestinal epithelial L-cells in response to meals and secreted into circulation. ${ }^{42}$ Circulating active GLP-1 induces insulin secretion in pancreatic $\beta$-cells and reduces glucagon secretion in pancreatic a-cells in a glucose-dependent manner. Since GLP-1 has a very short half-life, GLP-1 receptor agonists (GLP-1 RAs) were developed, and they showed a glucose-lowering effect by stimulating GLP-1 receptors. In addition to the glucose-lowering effect, GLP-1 RAs decrease appetite, delay gastric emptying, and induce weight loss. Therefore, GLP-1 RAs are an attractive candidate for the treatment of NAFLD, as they can reduce weight and enhance insulin action. However, according to the American Association for the Study of Liver Disease 2018 practice guideline, it is premature to consider GLP-1 RA for the treatment of liver disease in patients with NAFLD or NASH, due to limited evidence. ${ }^{28}$ However, several meta-analyses showed that GLP-1 RAs have additional roles in reducing the risk of major adverse CV events and CV deaths in high-risk patients with T2DM, especially in the Asian population. ${ }^{43}$ 


\section{Exenatide}

Exenatide is a synthetic form of a hormone isolated from Gila monster saliva, and the first agent of GLP-1 RA to be approved for the treatment of T2DM. In an observational pilot study, exenatide showed more qualitative ultrasonographic improvements in patients with NAFLD and T2DM compared with gliclazide, pioglitazone, sitagliptin, and liraglutide, although these differences were not significant. ${ }^{44}$ Several RCTs have found that exenatide alone or combined with other anti-diabetic drugs reduced liver enzymes, hepatic content, hepatic triglycerides, and epicardial fat. ${ }^{45-48}$ However, no well-designed placebo-controlled, doubleblind study with histology-proven outcomes has evaluated the effect of exenatide on NAFLD.

\section{Liraglutide}

Liraglutide is a long-acting GLP-1 RA, and is used for T2DM therapy at doses of up to $1.8 \mathrm{mg}$ daily. It has also been approved for obesity management at the dose of $3 \mathrm{mg}$ daily, and has been included in the largest number of studies on NAFLD among the GLP-1 RAs. Many studies showed that liraglutide reduced liver enzymes. ${ }^{49,50}$ In a meta-analysis of six RCTs conducted by the "Liraglutide Effect and Action in Diabetes (LEAD)" program, 26 weeks of liraglutide treatment (1.8 mg/day) improved ALT and hepatic steatosis in 4,442 patients with T2DM. ${ }^{49}$ However, these effects were likely mediated by weight loss and glycemic control, since they were lost after adjustment for weight reduction and HbA1c. In a prospective, single-center study (LIRA-NAFLD), liraglutide treatment (1.2 mg/day) for 6 months decreased liver fat content, as measured by proton magnetic resonance spectroscopy (MRS), in 68 patients with uncontrolled T2DM. ${ }^{51}$ The study also concluded that the effect of liver fat content reduction was mainly driven by body weight reduction. In a randomized trial which defined NAFLD as liver fat fraction $>5 \%$ on magnetic resonance imaging (MRI), liraglutide (3 mg/day) improved hepatic steatosis and hepatocellular apoptosis after 26 weeks in 30 obese patients; however, the benefits were not sustained after discontinuation, in contrast to lifestyle modification. ${ }^{52}$ Histological features were evaluated in two trials, LEAN (Liraglutide Safety and Efficacy in Patients With Non-Alcoholic Steatohepatitis) and LEAN-J (Liraglutide Effects in Non-Alcoholic Steatohepatitis and Non-Alcoholic Fatty Liver Disease With Glucose Intolerance in Japanese Patients). In the LEAN study, which was a randomized, placebo-controlled phase 2 trial consisting of 52 patients with biopsy-proven
NASH, 48 weeks of liraglutide (1.8 mg daily) was reported to resolve biopsy-proven NASH in nine out of 23 patients, whereas this was observed in only two out of 22 patients in the placebo group. ${ }^{53}$ In the LEAN-J study, which enrolled 19 Japanese patients with biopsy-proven NASH, repeated liver biopsy was performed in 10 subjects who continued liraglutide therapy ( $0.9 \mathrm{mg} /$ day) for 96 weeks. Seven subjects showed improved histological inflammation, six showed reduced liver fibrosis, and eight had improved NAS. $^{54}$ As expected, in both LEAN and LEAN-J studies, liraglutide was associated with significant reductions in body weight and BMI compared with placebo. However, their effects for NASH are not solely explained by the improvements in weight and metabolic phenotype. Liraglutide seems to be the most promising GLP-1 RA for NAFLD/NASH prevention and/or treatment, although large RCTs are needed to confirm its effects.

\section{Lixisenatide}

Similar to exenatide, lixsisenatide is based on exendin-4 with an addition of six lysines and deletion of a proline at the C-terminus. To date, there has been no RCT on lixisenatide for patients with NAFLD. In a meta-analysis of 12 RCTs on lixisenatide versus placebo and three RCTs with the active comparators, lixisenatide increased the proportion of patients with normalization of ALT compared with placebo or active comparators. ${ }^{55}$

\section{Dulaglutide}

Dulaglutide is a once-weekly GLP-1 RA and has some advantages, such as weekly injection, disposable and prefilled devices, as well as safety profiles similar to those of other GLP-1 RAs. The effect of dulaglutide on NAFLD is not well-known, as only a few studies have been conducted. In a post hoc analysis of AWARD (Assessment of Weekly Administration of LY2189265 [dulaglutide] in Diabetes) trials, dulaglutide (1.5 mg once weekly) improved liver enzymes compared with placebo in a pattern consistent with liver fat reduction. ${ }^{56}$ In a Japanese retrospective study, dulaglutide (0.75 mg once weekly) significantly improved liver enzymes and liver stiffness, as measured by transient elastography after 12 weeks of therapy in T2DM patients with biopsy-proven NASH. ${ }^{57}$

\section{Semaglutide}

Semaglutide is a human GLP-1 RA and has two forms, a onceweekly injectable agent and a once-daily oral agent. Currently, 
there is limited evidence regarding the effect of semaglutide on NAFLD. In a sub-analysis of the 104-week CV outcomes trial of T2DM (semaglutide 0.5 or $1.0 \mathrm{mg} /$ week) and a 52-week weight management trial (semaglutide $0.05-0.4 \mathrm{mg} /$ day), semaglutide significantly reduced ALT in subjects with T2DM ${ }^{58}$ To investigate the safety and efficacy of semaglutide (once-daily subcutaneous injection) on NASH (stage 1-3 fibrosis), a phase 2, randomized, double-blind, placebo-controlled trial (SEMA-NASH study, NCT02970942) was initiated, the final results of which are currently pending. However, there is no existing data regarding the effects of an oral form of semaglutide on NAFLD.

\section{Tirzepatide}

Tirzepatide is a novel unimolecular, multi-functional peptide that combines GLP-1 and glucose-dependent insulinotropic polypeptide (GIP) receptor agonist activities. GIP is an incretin secreted from $\mathrm{K}$ cells in the upper small intestine in response to food. Unlike GLP-1, GIP is both glucagonotropic and insulinotropic in a glucose-dependent manner, dose-dependently stimulating glucagon secretion under hypoglycemic conditions and insulin under hyperglycemic conditions. ${ }^{59}$ The number of studies conducted to demonstrate the effect of tirzepatide on NAFLD is very limited. Patients with T2DM received either once weekly tirzepatide (1, 5, 10 , or $15 \mathrm{mg})$, dulaglutide $(1.5 \mathrm{mg})$, or placebo for 26 weeks. In post hoc analyses, significant $(P<0.05)$ reductions from baseline in ALT (all groups), aspartate aminotransferase (AST) (all groups except tirzepatide $10 \mathrm{mg}$ ), keratin-18 (tirzepatide 5, 10, $15 \mathrm{mg}$ ), and procollagen III (tirzepatide $15 \mathrm{mg}$ ) were observed at 26 weeks. ${ }^{60}$ Although one study cannot provide sufficient evidence of efficacy for tirzepatide in subjects with NASH, weight reduction along with changes in NASH biomarker suggest the need for further trials of tirzepatide in subjects with NASH.

\section{DIPEPTIDYL PEPTIDASE-4 (DPP-4) INHIBITORS}

DPP-4 inhibitors demonstrate a glucose-lowering effect by increasing the incretin effect through the delay of quick inactivation of GLP-1 in plasma. Several studies found that serum DPP-4 level was elevated in NASH patients, and also correlated with hepatic steatosis, fibrosis, and hepatocyte apoptosis. ${ }^{61,62}$ These findings support the notion that DPP-4 inhibitors may improve the histological features of NAFLD/NASH; however, there has been only a few studies demonstrating the significant efficacy of DPP-4 inhibi- tors in patients with NAFLD. DPP-4 inhibitors are not yet believed to have a beneficial effect on NAFLD.

\section{Sitagliptin}

As a first-in-class DPP-4 inhibitor, sitagliptin has been most widely studied to evaluate its efficacy on NAFLD/NASH. However, sitagliptin has shown conflicting evidence regarding its effects on NAFLD. Sitagliptin treatment (50 mg/day) for 4 months improved liver enzymes in patients with NAFLD $(n=30)$ diagnosed by ultrasound..$^{63}$ In an observational pilot study of 15 T2DM patients with biopsy-proven NASH, sitagliptin treatment (100 mg/day) for 1 year ameliorated liver enzymes, hepatocyte ballooning, and NASH scores. ${ }^{64}$ In a prospective, 24-week, single-center, open-label comparative study that enrolled 20 Japanese patients with T2DM and NAFLD, sitagliptin significantly reduced intrahepatic lipid content, as assessed by 1) H-MRS, and total body fat mass after 24 weeks. ${ }^{65}$ On the other hand, in 44 patients with T2DM and biopsy-proven NAFLD, sitagliptin treatment ( $50 \mathrm{mg} /$ day) for 12 months did not reduce liver enzymes, although HbA1c was reduced by $0.7 \% .{ }^{66}$ In a RCT that enrolled 50 NAFLD patients, liver enzymes and magnetic resonance elastography-derived liver stiffness were not improved by 24 weeks of sitagliptin treatment (100 $\mathrm{mg} / \mathrm{day}){ }^{67}$ Moreover, in many existing studies, sitagliptin treatment failed to produce any reduction in liver fat content and liver enzymes ${ }^{68,69}$ Based on previous clinical trials, sitagliptin is not yet believed to have a beneficial effect on NAFLD.

\section{Vildagliptin}

A few trials have demonstrated that vildagliptin has an effect on NAFLD. In 44 patients with T2DM and hepatic steatosis, vildagliptin treatment (50 mg twice daily) for 6 months decreased liver enzymes and intrahepatic triglyceride content, as assessed by MRI. ${ }^{70}$ In a RCT conducted in Pakistan, vildagliptin (50 mg twice daily) for 12 weeks improved liver enzymes and steatosis grading, as assessed by ultrasound. ${ }^{11}$

\section{Other DPP-4 inhibitors}

Not many studies have been conducted to evaluate the efficacy of other DPP-4 inhibitors, including gemigliptin, alogliptin, teneligliptin, saxagliptin, anagliptin, and linagliptin, on NAFLD. In an animal model, many DPP-4 inhibitors, including gemigliptin, alogliptin, teneligliptin, and linagliptin, were able to alleviate hepatic 
steatosis and hepatic lipogenesis. ${ }^{72-75}$ In human clinical trials, 12 months of alogliptin treatment ( $25 \mathrm{mg} /$ day) in NAFLD patients with T2DM yielded a decrease in NAFLD progression. ${ }^{76}$ With saxagliptin, liver enzymes and hepatic steatosis were improved after treatment for 24 weeks in 95 patients with NAFLD and T2DM. ${ }^{77}$

\section{SODIUM-GLUCOSE COTRANSPORTER 2 (SGLT2) INHIBITORS}

SGLT2 is responsible for a majority of the glucose reabsorption that occurs in proximal renal tubules. SGLT2 inhibitors are a novel class of anti-diabetic drugs that improve hyperglycemia by inhibiting urinary glucose reabsorption from the kidney. ${ }^{78}$ In addition to a glucose-lowering effect, SGLT2 inhibitors exert several cardiorenal and metabolic benefits, including weight loss; therefore, they are thought to be promising therapeutic agents for patients with NAFLD. ${ }^{79}$ Although beneficial effects continue to be reported, SGLT2 inhibitors are not yet generally recommended for the treatment of NAFLD.

\section{Dapagliflozin}

Many small-scale studies have shown that dapagliflozin treatment improved liver enzymes, liver fat content, or liver stiffness in patients with NAFLD. ${ }^{80,81}$ In a single-arm, nonrandomized, openlabel study, dapagliflozin treatment ( $5 \mathrm{mg} /$ day) for 24 weeks reduced liver enzymes and metabolic parameters in 11 patients with biopsy-proven NASH. ${ }^{82}$ The Effects of Omega-3 Carboxylic Acids and Dapagliflozin on Liver Fat Content in Diabetic Patients (EFFECT-II) study found that dapagliflozin (10 mg/day) combined with omega-3 carboxylic acids reduced liver fat content, as assessed by MRI-derived proton density fat fraction (MRI-PDFF), and dapagliflozin monotherapy reduced all measured hepatocyte injury markers in patients with T2DM and NAFLD. ${ }^{83}$ In a randomized, active-controlled, open-label trial, dapagliflozin treatment (5 $\mathrm{mg} /$ day) for 24 weeks improved liver enzymes, steatosis, and fibrosis, as measured by transient elastography (FibroScan), in 57 patients with T2DM and NAFLD, as defined by ultrasound. ${ }^{84}$ Furthermore, the Dapagliflozin Efficacy and Action in NASH (DEAN) study (NCT03723252) is still ongoing, with the objective to assess the efficacy and safety of dapagliflozin for improving biopsy-proven NASH and metabolic risk factors. This study is a phase 3, multicenter, randomized, double-blind, placebo-controlled trial, and will enroll 100 participants until November 2021.

\section{Canagliflozin}

Several studies have evaluated the efficacy of canagliflozin on NAFLD. ${ }^{85-88}$ A meta-analysis of 11 trials with a total of 6,745 T2DM patients reported that canagliflozin significantly decreased liver enzymes. ${ }^{85}$ In a RCT, canagliflozin (300 mg/day) for 24 weeks reduced liver enzymes and intrahepatic triglyceride content, as measured by proton-MRS, in 37 patients with T2DM and NAFLD. ${ }^{86}$ Likewise, in a small $(n=20)$, prospective, non-randomized, openlabel, single-arm study, canagliflozin (100 mg daily) treatment for 12 months significantly reduced hepatic fat fraction, as measured by MRI, in patients with T2DM and NAFLD. ${ }^{87}$ In nine patients with T2DM and biopsy-proven NASH, canagliflozin treatment (100 $\mathrm{mg} /$ day) for 24 weeks improved the histological features of NASH, including steatosis, lobular inflammation, ballooning, and fibrosis stage. $^{88}$

\section{Empagliflozin}

Only a few studies have evaluated the efficacy of empagliflozin on NAFLD. In the Empagliflozin Cardiovascular Outcome Event Trial in Type 2 Diabetes Mellitus Patients (EMPA-REG OUTCOME), empagliflozin reduced liver enzymes (ALT>AST) in patients with T2DM. $^{89}$ A single-center, retrospective, observational study found that empagliflozin ( $5 \mathrm{mg} /$ day) for 6 months significantly improved liver enzymes in 46 patients with T2DM. ${ }^{90}$ The Effect of Empagliflozin on Liver Fat Content in Patients With Type 2 Diabetes ( $E$ LIFT) trial, an investigator-initiated, prospective, open-label, randomized clinical study involving 50 patients with T2DM and NAFLD, showed that empagliflozin (10 mg daily) for 20 weeks significantly reduced liver enzymes and liver fat, as measured by MRI-PDFF. ${ }^{91}$

\section{Ipragliflozin}

Ipragliflozin is used in several countries, including South Korea and Japan. In 43 patients with T2DM and NAFLD, ipragliflozin treatment for 24 weeks (50 mg daily) reduced liver enzymes. ${ }^{92}$ Despite the small number of studies, ipragliflozin showed similar beneficial effects to pioglitazone on NAFLD. The open-label, randomized, active-controlled trial compared the efficacy of ipragliflozin (50 mg daily) versus pioglitazone (15-30 mg daily) in 66 patients with T2DM and NAFLD. At week 24, ipragliflozin exerted equally beneficial effects on NAFLD compared with pioglitazone. ${ }^{93}$ Furthermore, ipragliflozin (50 mg daily) treatment for 24 weeks 
significantly ameliorated liver steatosis and reduced excessive fat in 44 euglycemic patients with T2DM and NAFLD who were taking metformin and pioglitazone at baseline. ${ }^{94}$

\section{Other SGLT2 inhibitors}

Several pre-clinical studies conducted with animal experimental models have shown that luseogliflozin and remogliflozin could be associated with improvement in hepatic steatosis. ${ }^{95,96}$ In 32 patients with T2DM and NAFLD diagnosed by abdominal computed tomography or ultrasound, luseogliflozin treatment (2.5 $\mathrm{mg}$ daily) for 6 months reduced liver fat deposition compared to metformin. ${ }^{97} \mathrm{~A}$ prospective, single-arm trial (LEAD trial) showed that luseogliflozin treatment ( $2.5 \mathrm{mg}$ daily) for 24 weeks reduced hepatic fat content, as evaluated by MRI, in patients with T2DM and NAFLD. ${ }^{98}$

\section{a-GLUCOSIDASE INHIBITORS}

a-glucosidase inhibitors, including acarbose, miglitol, and voglibose, are oral glucose-lowering agents that specifically inhibit a-glucosidases in the brush border of the small intestine to prevent digestion of carbohydrates, such as starch and sugar. To date, only a limited number of studies have investigated the effect of a-glucosidase inhibitors on NAFLD. In a mouse model of NAFLD, combination therapy with ezetimibe and acarbose for 24 weeks improved the histopathological findings. ${ }^{99}$ Administration of miglitol (150 mg/day) for 12 months in 17 T2DM patients with histologically-confirmed NASH showed significantly decreased BMI and serum ALT. ${ }^{100}$ Post-treatment liver biopsy of 11 patients revealed significant improvements in steatosis, lobular inflammation, portal inflammation scores, and NAFLD activity scores (from $5.5 \pm 1.5$ to $3.9 \pm 1.4, P=0.012$ ). However, fibrosis and hepatocyte ballooning scores remained unchanged.

\section{SULFONYLUREAS AND MEGLITINIDES}

Insulin resistance with hyperinsulinemia might be deleterious to the liver and associated with high intrahepatic triglyceride content. ${ }^{101}$ Sulfonylureas and meglitinides are insulin secretagogues that lower glucose level by enhancing insulin secretion from pancreatic $\beta$-cells. Metformin and pioglitazone may be safely used in patients with NAFLD, whereas sulfonylureas and insulin itself have been associated with NAFLD progression and adverse outcomes. ${ }^{102}$ This class of drugs has rarely been studied regarding its impact on NAFLD.

\section{DRUGS UNDER INVESTIGATION}

Various types of promising therapeutic targets for T2DM are under investigation. Protein tyrosine phosphatase $1 \mathrm{~B}$ inhibitor, free fatty acid receptors 1 , aldose reductase, glycogen phosphorylase, fructose-1, 6-bisphosphatase, glucagon receptor antagonist, and phosphoenolpyruvate carboxykinase are currently being assessed. ${ }^{103}$ Among them, drugs that show weight reduction can be effective in the treatment of NAFLD. Further studies are warranted to evaluate the efficacy of novel anti-diabetic drugs for NAFLD.

\section{CONCLUSIONS}

To this day, the only approved treatment option for NAFLD is weight reduction, although much progress has been made in the understanding, diagnosis, and treatment of NAFLD over past decades. Since T2DM and NAFLD have a partly shared pathophysiology that includes insulin resistance even genetically, ${ }_{1}^{104-106}$ anti-diabetic drugs are a good candidate for the treatment of NAFLD. Most anti-diabetic drugs showed an improvement in liver enzymes, but there is limited evidence of any beneficial effect on histological outcomes. Among anti-diabetic drugs, pioglitazone has the strongest evidence for a role in NAFLD treatment, and it has shown improvement in liver histology for patients with biopsy-proven NASH. Liraglutide has also shown promising evidence, although it is premature to consider a GLP-1 RA for the treatment of liver disease in patients with NAFLD. Further well-designed RCTs should be conducted to determine the role of these anti-diabetic drugs in NAFLD treatment.

\section{Authors' contribution}

Kyung-Soo Kim and Byung-Wan Lee contributed to the literature review and manuscript preparation.

\section{Acknowledgements}

The authors thank Medical Illustration \& Design, part of the Medical Research Support Services of Yonsei University College of Medicine, for all artistic support related to this work. 


\section{Conflicts of Interest}

The authors have no conflicts to disclose.

\section{REFERENCES}

1. Younossi Z, Stepanova M, Ong JP, Jacobson IM, Bugianesi $E$, Duseja A, et al. Nonalcoholic steatohepatitis is the fastest growing cause of hepatocellular carcinoma in liver transplant candidates. Clin Gastroenterol Hepatol 2019;17:748-755.e3.

2. Byrne CD, Targher G. NAFLD: a multisystem disease. J Hepatol 2015;62(1 Suppl):S47-S64.

3. Kim KS, Lee BW, Kim YJ, Lee DH, Cha BS, Park CY. Nonalcoholic fatty liver disease and diabetes: part II: treatment. Diabetes Metab J 2019:43:127-143.

4. Rhee EJ. Nonalcoholic fatty liver disease and diabetes: an epidemiological perspective. Endocrinol Metab (Seoul) 2019;34:226-233.

5. Vernon G, Baranova A, Younossi ZM. Systematic review: the epidemiology and natural history of non-alcoholic fatty liver disease and non-alcoholic steatohepatitis in adults. Aliment Pharmacol Ther 2011;34:274-285.

6. Lee SB, Kim MK, Kang S, Park K, Kim JH, Baik SJ, et al. Triglyceride glucose index is superior to the homeostasis model assessment of insulin resistance for predicting nonalcoholic fatty liver disease in Korean adults. Endocrinol Metab (Seoul) 2019;34:179-186.

7. Arrese M, Barrera F, Triantafilo N, Arab JP. Concurrent nonalcoholic fatty liver disease and type 2 diabetes: diagnostic and therapeutic considerations. Expert Rev Gastroenterol Hepatol 2019;13:849866.

8. Xia MF, Bian H, Gao X. NAFLD and diabetes: two sides of the same coin? Rationale for gene-based personalized NAFLD treatment. Front Pharmacol 2019;10:877.

9. Lee $Y H$, Cho Y, Lee BW, Park CY, Lee DH, Cha BS, et al. Nonalcoholic fatty liver disease in diabetes. Part I: epidemiology and diagnosis. Diabetes Metab J 2019:43:31-45.

10. Fracanzani AL, Valenti L, Bugianesi $E$, Andreoletti M, Colli A, Vanni $E$, et al. Risk of severe liver disease in nonalcoholic fatty liver disease with normal aminotransferase levels: a role for insulin resistance and diabetes. Hepatology 2008;48:792-798.

11. Ulasoglu C, Enc FY, Kaya E, Yilmaz Y. Characterization of patients with biopsy-proven non-alcoholic fatty liver disease and normal aminotransferase levels. J Gastrointestin Liver Dis 2019;28:427431.

12. Kim SK, Choi YJ, Huh BW, Park SW, Lee EJ, Cho YW, et al. Nonalcoholic fatty liver disease is associated with increased carotid intima-media thickness only in type 2 diabetic subjects with insulin resistance. J Clin Endocrinol Metab 2014;99:1879-1884.
13. Kim BY, Jung CH, Mok JO, Kang SK, Kim CH. Prevalences of diabetic retinopathy and nephropathy are lower in Korean type 2 diabetic patients with non-alcoholic fatty liver disease. J Diabetes Investig 2014;5:170-175.

14. Younossi ZM, Golabi P, de Avila L, Paik JM, Srishord M, Fukui N, et al. The global epidemiology of NAFLD and NASH in patients with type 2 diabetes: a systematic review and meta-analysis. J Hepatol 2019;71:793-801.

15. European Association for the Study of the Liver (EASL); European Association for the Study of Diabetes (EASD); European Association for the Study of Obesity (EASO). EASL-EASD-EASO clinical practice guidelines for the management of non-alcoholic fatty liver disease. J Hepatol 2016;64:1388-1402.

16. Jang JE, Cho Y, Lee BW, Shin ES, Lee SH. Effectiveness of exercise intervention in reducing body weight and glycosylated hemoglobin levels in patients with type 2 diabetes mellitus in Korea: a systematic review and meta-analysis. Diabetes Metab J 2019;43:302-318.

17. Sanchez-Rangel E, Inzucchi SE. Metformin: clinical use in type 2 diabetes. Diabetologia 2017;60:1586-1593.

18. Rena G, Hardie DG, Pearson ER. The mechanisms of action of metformin. Diabetologia 2017;60:1577-1585.

19. Kim MK, Ko SH, Kim BY, Kang ES, Noh J, Kim SK, et al. 2019 clinical practice guidelines for type 2 diabetes mellitus in Korea. Diabetes Metab J 2019;43:398-406.

20. Kang YM, Jung CH, Lee SH, Kim SW, Song KH, Kim SG, et al. Effectiveness and safety of adding basal insulin glargine in patients with type 2 diabetes mellitus exhibiting inadequate response to metformin and dpp-4 inhibitors with or without sulfonylurea. Diabetes Metab J 2019;43:432-446.

21. Lin HZ, Yang SQ, Chuckaree C, Kuhajda F, Ronnet G, Diehl AM. Metformin reverses fatty liver disease in obese, leptin-deficient mice. Nat Med 2000;6:998-1003.

22. Song YM, Lee YH, Kim JW, Ham DS, Kang ES, Cha BS, et al. Metformin alleviates hepatosteatosis by restoring SIRT1-mediated autophagy induction via an AMP-activated protein kinase-independent pathway. Autophagy 2015;11:46-59.

23. Marchesini G, Brizi M, Bianchi G, Tomassetti S, Zoli M, Melchionda N. Metformin in non-alcoholic steatohepatitis. Lancet 2001;358:893-894.

24. Lavine JE, Schwimmer JB, Van Natta ML, Molleston JP, Murray KF, Rosenthal $P$, et al. Effect of vitamin $E$ or metformin for treatment of nonalcoholic fatty liver disease in children and adolescents: the TONIC randomized controlled trial. JAMA 2011;305:1659-1668.

25. Rakoski MO, Singal AG, Rogers MA, Conjeevaram H. Meta-analysis: insulin sensitizers for the treatment of non-alcoholic steatohepatitis. Aliment Pharmacol Ther 2010;32:1211-1221.

26. Musso G, Gambino R, Cassader M, Pagano G. A meta-analysis of randomized trials for the treatment of nonalcoholic fatty liver dis- 
ease. Hepatology 2010;52:79-104.

27. Li Y, Liu L, Wang B, Wang J, Chen D. Metformin in non-alcoholic fatty liver disease: a systematic review and meta-analysis. Biomed Rep 2013;1:57-64.

28. Chalasani N, Younossi Z, Lavine JE, Charlton M, Cusi K, Rinella M, et al. The diagnosis and management of nonalcoholic fatty liver disease: practice guidance from the American Association for the Study of Liver Diseases. Hepatology 2018;67:328-357.

29. Cunha V, Cotrim HP, Rocha R, Carvalho K, Lins-Kusterer L. Metformin in the prevention of hepatocellular carcinoma in diabetic patients: a systematic review. Ann Hepatol 2020;19:232-237.

30. Promrat K, Lutchman G, Uwaifo GI, Freedman RJ, Soza A, Heller T, et al. A pilot study of pioglitazone treatment for nonalcoholic steatohepatitis. Hepatology 2004;39:188-196.

31. Belfort R, Harrison SA, Brown K, Darland C, Finch J, Hardies J, et al. A placebo-controlled trial of pioglitazone in subjects with nonalcoholic steatohepatitis. N Engl J Med 2006;355:2297-2307.

32. He L, Liu X, Wang L, Yang Z. Thiazolidinediones for nonalcoholic steatohepatitis: a meta-analysis of randomized clinical trials. Medicine (Baltimore) 2016;95:e4947.

33. Singh S, Khera R, Allen AM, Murad MH, Loomba R. Comparative effectiveness of pharmacological interventions for nonalcoholic steatohepatitis: a systematic review and network meta-analysis. Hepatology 2015;62:1417-1432.

34. Sanyal AJ, Chalasani N, Kowdley KV, McCullough A, Diehl AM, Bass NM, et al. Pioglitazone, vitamin $\mathrm{E}$, or placebo for nonalcoholic steatohepatitis. N Engl J Med 2010;362:1675-1685.

35. Cusi K, Orsak B, Bril F, Lomonaco R, Hecht J, Ortiz-Lopez C, et al. Long-term pioglitazone treatment for patients with nonalcoholic steatohepatitis and prediabetes or type 2 diabetes mellitus: a randomized trial. Ann Intern Med 2016;165:305-315.

36. Musso G, Cassader M, Paschetta E, Gambino R. Thiazolidinediones and advanced liver fibrosis in nonalcoholic steatohepatitis: a metaanalysis. JAMA Intern Med 2017;177:633-640.

37. logna Prat L, Tsochatzis EA. The effect of antidiabetic medications on non-alcoholic fatty liver disease (NAFLD). Hormones (Athens) 2018:17:219-229.

38. Kim SG, Kim DM, Woo JT, Jang HC, Chung CH, Ko KS, et al. Efficacy and safety of lobeglitazone monotherapy in patients with type 2 diabetes mellitus over 24-weeks: a multicenter, randomized, double-blind, parallel-group, placebo controlled trial. PLoS One 2014;9:e92843.

39. Choung S, Joung KH, You BR, Park SK, Kim HJ, Ku BJ. Treatment with lobeglitazone attenuates hepatic steatosis in diet-induced obese mice. PPAR Res 2018;2018:4292509.

40. Lee YS, Park JS, Lee DH, Lee DK, Kwon SW, Lee BW, et al. The antidiabetic drug lobeglitazone protects mice from lipogenesisinduced liver injury via mechanistic target of rapamycin complex 1 inhibition. Front Endocrinol (Lausanne) 2018;9:539.

41. Lee YH, Kim JH, Kim SR, Jin HY, Rhee EJ, Cho YM, et al. Lobeglitazone, a novel thiazolidinedione, improves non-alcoholic fatty liver disease in type 2 diabetes: its efficacy and predictive factors related to responsiveness. J Korean Med Sci 2017;32:60-69.

42. Baggio LL, Drucker DJ. Biology of incretins: GLP-1 and GIP. Gastroenterology 2007;132:2131-2157.

43. Kang YM, Cho YK, Lee J, Lee SE, Lee WJ, Park JY, et al. Asian subpopulations may exhibit greater cardiovascular benefit from longacting glucagon-like peptide 1 receptor agonists: a meta-analysis of cardiovascular outcome trials. Diabetes Metab J 2019;43:410421.

44. García Díaz E, Guagnozzi D, Gutiérrez V, Mendoza C, Maza C, Larrañaga $Y$, et al. Effect of incretin therapies compared to pioglitazone and gliclazide in non-alcoholic fatty liver disease in diabetic patients not controlled on metformin alone: an observational, pilot study. Endocrinol Nutr 2016;63:194-201.

45. Sathyanarayana P, Jogi M, Muthupillai R, Krishnamurthy R, Samson $S L$, Bajaj M. Effects of combined exenatide and pioglitazone therapy on hepatic fat content in type 2 diabetes. Obesity (Silver Spring) 2011;19:2310-2315.

46. Blaslov K, Zibar K, Bulum T, Duvnjak L. Effect of exenatide therapy on hepatic fat quantity and hepatic biomarkers in type 2 diabetic patients. Clin Res Hepatol Gastroenterol 2014;38:e61-e63.

47. Dutour A, Abdesselam I, Ancel P, Kober F, Mrad G, Darmon P, et al. Exenatide decreases liver fat content and epicardial adipose tissue in patients with obesity and type 2 diabetes: a prospective randomized clinical trial using magnetic resonance imaging and spectroscopy. Diabetes Obes Metab 2016;18:882-891.

48. Bi $Y$, Zhang $B, X u W$, Yang $H$, Feng $W$, Li $C$, et al. Effects of exenatide, insulin, and pioglitazone on liver fat content and body fat distributions in drug-naive subjects with type 2 diabetes. Acta Diabetol 2014;51:865-873.

49. Armstrong MJ, Houlihan DD, Rowe IA, Clausen WH, Elbrond B, Gough SC, et al. Safety and efficacy of liraglutide in patients with type 2 diabetes and elevated liver enzymes: individual patient data meta-analysis of the LEAD program. Aliment Pharmacol Ther 2013:37:234-242.

50. Tian F, Zheng Z, Zhang D, He S, Shen J. Efficacy of liraglutide in treating type 2 diabetes mellitus complicated with non-alcoholic fatty liver disease. Biosci Rep 2018;38:BSR20181304.

51. Petit JM, Cercueil JP, Loffroy R, Denimal D, Bouillet B, Fourmont C, et al. Effect of liraglutide therapy on liver fat content in patients with inadequately controlled type 2 diabetes: the Lira-NAFLD Study. J Clin Endocrinol Metab 2017;102:407-415.

52. Khoo J, Hsiang JC, Taneja R, Koo SH, Soon GH, Kam CJ, et al. Randomized trial comparing effects of weight loss by liraglutide with lifestyle modification in non-alcoholic fatty liver disease. Liver Int 


\section{9:39:941-949.}

53. Armstrong MJ, Gaunt P, Aithal GP, Barton D, Hull D, Parker R, et al. Liraglutide safety and efficacy in patients with non-alcoholic steatohepatitis (LEAN): a multicentre, double-blind, randomised, placebo-controlled phase 2 study. Lancet 2016;387:679-690.

54. Eguchi Y, Kitajima Y, Hyogo H, Takahashi H, Kojima M, Ono M, et al. Pilot study of liraglutide effects in non-alcoholic steatohepatitis and non-alcoholic fatty liver disease with glucose intolerance in Japanese patients (LEAN-J). Hepatol Res 2015;45:269-278.

55. Gluud LL, Knop FK, Vilsbøll T. Effects of lixisenatide on elevated liver transaminases: systematic review with individual patient data meta-analysis of randomised controlled trials on patients with type 2 diabetes. BMJ Open 2014;4:e005325.

56. Cusi K, Sattar N, García-Pérez LE, Pavo I, Yu M, Robertson KE, et al. Dulaglutide decreases plasma aminotransferases in people with type 2 diabetes in a pattern consistent with liver fat reduction: a post hoc analysis of the AWARD programme. Diabet Med 2018;35:1434-1439.

57. Seko Y, Sumida Y, Tanaka S, Mori K, Taketani H, Ishiba H, et al. Effect of 12-week dulaglutide therapy in Japanese patients with biopsy-proven non-alcoholic fatty liver disease and type 2 diabetes mellitus. Hepatol Res 2017;47:1206-1211.

58. Newsome P, Francque S, Harrison S, Ratziu V, Van Gaal L, Calanna S, et al. Effect of semaglutide on liver enzymes and markers of inflammation in subjects with type 2 diabetes and/or obesity. Aliment Pharmacol Ther 2019;50:193-203.

59. Coskun T, Sloop KW, Loghin C, Alsina-Fernandez J, Urva S, Bokvist KB, et al. LY3298176, a novel dual GIP and GLP-1 receptor agonist for the treatment of type 2 diabetes mellitus: from discovery to clinical proof of concept. Mol Metab 2018;18:3-14.

60. Hartman ML, Sanyal AJ, Loomba R, Wilson JM, Nikooienejad A, Bray $R$, et al. Effects of novel dual GIP and GLP-1 receptor agonist tirzepatide on biomarkers of nonalcoholic steatohepatitis in patients with type 2 diabetes. Diabetes Care 2020;43:1352-1355.

61. Balaban YH, Korkusuz P, Simsek H, Gokcan H, Gedikoglu G, Pinar A, et al. Dipeptidyl peptidase IV (DDP IV) in NASH patients. Ann Hepatol 2007;6:242-250.

62. Wang $X$, Hausding M, Weng SY, Kim YO, Steven S, Klein T, et al. Gliptins suppress inflammatory macrophage activation to mitigate inflammation, fibrosis, oxidative stress, and vascular dysfunction in models of nonalcoholic steatohepatitis and liver fibrosis. Antioxid Redox Signal 2018;28:87-109.

63. Iwasaki T, Yoneda M, Inamori M, Shirakawa J, Higurashi T, Maeda S, et al. Sitagliptin as a novel treatment agent for non-alcoholic fatty liver disease patients with type 2 diabetes mellitus. Hepatogastroenterology 2011;58:2103-2105.

64. Yilmaz Y, Yonal O, Deyneli O, Celikel CA, Kalayci C, Duman DG. Effects of sitagliptin in diabetic patients with nonalcoholic steato- hepatitis. Acta Gastroenterol Belg 2012;75:240-244.

65. Kato H, Nagai Y, Ohta A, Tenjin A, Nakamura Y, Tsukiyama $H$, et al. Effect of sitagliptin on intrahepatic lipid content and body fat in patients with type 2 diabetes. Diabetes Res Clin Pract 2015;109:199205.

66. Fukuhara $T$, Hyogo $H$, Ochi $H$, Fujino $H$, Kan $H$, Naeshiro $N$, et al. Efficacy and safety of sitagliptin for the treatment of nonalcoholic fatty liver disease with type 2 diabetes mellitus. Hepatogastroenterology 2014;61:323-328.

67. Cui J, Philo L, Nguyen P, Hofflich H, Hernandez C, Bettencourt R, et al. Sitagliptin vs. placebo for non-alcoholic fatty liver disease: a randomized controlled trial. J Hepatol 2016;65:369-376.

68. Watanabe T, Tamura Y, Kakehi S, Funayama T, Gastaldelli A, Takeno $K$, et al. Effects of sitagliptin on ectopic fat contents and glucose metabolism in type 2 diabetic patients with fatty liver: a pilot study. J Diabetes Investig 2015;6:164-172.

69. Joy TR, McKenzie CA, Tirona RG, Summers K, Seney S, Chakrabarti $S$, et al. Sitagliptin in patients with non-alcoholic steatohepatitis: a randomized, placebo-controlled trial. World J Gastroenterol 2017;23:141-150.

70. Macauley M, Hollingsworth KG, Smith FE, Thelwall PE, Al-Mrabeh A, Schweizer A, et al. Effect of vildagliptin on hepatic steatosis. J Clin Endocrinol Metab 2015;100:1578-1585.

71. Hussain M, Majeed Babar MZ, Hussain MS, Akhtar L. Vildagliptin ameliorates biochemical, metabolic and fatty changes associated with non alcoholic fatty liver disease. Pak J Med Sci 2016;32:13961401.

72. Hwang HJ, Jung TW, Kim BH, Hong HC, Seo JA, Kim SG, et al. A dipeptidyl peptidase-IV inhibitor improves hepatic steatosis and insulin resistance by AMPK-dependent and JNK-dependent inhibition of LECT2 expression. Biochem Pharmacol 2015;98:157-166.

73. Tobita H, Sato S, Yazaki T, Mishiro T, Ishimura N, Ishihara S, et al. Alogliptin alleviates hepatic steatosis in a mouse model of nonalcoholic fatty liver disease by promoting CPT1a expression via Thr172 phosphorylation of AMPKa in the liver. Mol Med Rep 2018;17:6840-6846.

74. Ideta T, Shirakami Y, Miyazaki T, Kochi T, Sakai H, Moriwaki H, et al. The dipeptidyl peptidase-4 inhibitor teneligliptin attenuates hepatic lipogenesis via AMPK activation in non-alcoholic fatty liver disease model mice. Int J Mol Sci 2015;16:29207-29218.

75. Klein T, Fujii M, Sandel J, Shibazaki Y, Wakamatsu K, Mark M, et al. Linagliptin alleviates hepatic steatosis and inflammation in a mouse model of non-alcoholic steatohepatitis. Med Mol Morphol 2014:47:137-149.

76. Mashitani T, Noguchi R, Okura Y, Namisaki T, Mitoro A, Ishii H, et al. Efficacy of alogliptin in preventing non-alcoholic fatty liver disease progression in patients with type 2 diabetes. Biomed Rep 2016;4:183-187. 
77. Li JJ, Zhang P, Fan B, Guo XL, Zheng ZS. The efficacy of saxagliptin in T2DM patients with non-alcoholic fatty liver disease: preliminary data. Rev Assoc Med Bras (1992) 2019;65:33-37.

78. Hwang YC, Kim JH, Lee BW, Lee WJ. A lower baseline urinary glucose excretion predicts a better response to the sodium glucose cotransporter 2 inhibitor. Diabetes Metab J 2019;43:898-905.

79. Kim GS, Park JH, Won JC. The role of glucagon-like peptide 1 receptor agonists and sodium-glucose cotransporter 2 inhibitors in reducing cardiovascular events in patients with type 2 diabetes. Endocrinol Metab (Seoul) 2019;34:106-116.

80. Choi DH, Jung CH, Mok JO, Kim CH, Kang SK, Kim BY. Effect of dapagliflozin on alanine aminotransferase improvement in type 2 diabetes mellitus with non-alcoholic fatty liver disease. Endocrinol Metab (Seoul) 2018:33:387-394.

81. Kurinami N, Sugiyama S, Yoshida A, Hieshima K, Miyamoto F, Kajiwara $K$, et al. Dapagliflozin significantly reduced liver fat accumulation associated with a decrease in abdominal subcutaneous fat in patients with inadequately controlled type 2 diabetes mellitus. Diabetes Res Clin Pract 2018;142:254-263.

82. Tobita H, Sato S, Miyake T, Ishihara S, Kinoshita Y. Effects of dapagliflozin on body composition and liver tests in patients with nonalcoholic steatohepatitis associated with type 2 diabetes mellitus: a prospective, open-label, uncontrolled study. Curr Ther Res Clin Exp 2017;87:13-19.

83. Eriksson JW, Lundkvist $P$, Jansson PA, Johansson L, Kvarnström $M$, Moris $L$, et al. Effects of dapagliflozin and N-3 carboxylic acids on non-alcoholic fatty liver disease in people with type 2 diabetes: a double-blind randomised placebo-controlled study. Diabetologia 2018;61:1923-1934.

84. Shimizu M, Suzuki K, Kato K, Jojima T, lijima T, Murohisa T, et al. Evaluation of the effects of dapagliflozin, a sodium-glucose cotransporter-2 inhibitor, on hepatic steatosis and fibrosis using transient elastography in patients with type 2 diabetes and nonalcoholic fatty liver disease. Diabetes Obes Metab 2019;21:285292.

85. Li B, Wang Y, Ye Z, Yang H, Cui X, Wang Z, et al. Effects of canagliflozin on fatty liver indexes in patients with type 2 diabetes: a meta-analysis of randomized controlled trials. J Pharm Pharm Sci 2018;21:222-235.

86. Cusi K, Bril F, Barb D, Polidori D, Sha S, Ghosh A, et al. Effect of canagliflozin treatment on hepatic triglyceride content and glucose metabolism in patients with type 2 diabetes. Diabetes Obes Metab 2019;21:812-821.

87. Inoue M, Hayashi A, Taguchi T, Arai R, Sasaki S, Takano K, et al. Effects of canagliflozin on body composition and hepatic fat content in type 2 diabetes patients with non-alcoholic fatty liver disease. J Diabetes Investig 2019;10:1004-1011.

88. Akuta N, Kawamura Y, Watanabe C, Nishimura A, Okubo M,
Mori Y, et al. Impact of sodium glucose cotransporter 2 inhibitor on histological features and glucose metabolism of non-alcoholic fatty liver disease complicated by diabetes mellitus. Hepatol Res 2019:49:531-539.

89. Sattar N, Fitchett D, Hantel S, George JT, Zinman B. Empagliflozin is associated with improvements in liver enzymes potentially consistent with reductions in liver fat: results from randomised trials including the EMPA-REG OUTCOME ${ }^{\circ}$ trial. Diabetologia 2018:61:2155-2163.

90. Lee PCH, Gu Y, Yeung MY, Fong CHY, Woo YC, Chow WS, et al. Dapagliflozin and empagliflozin ameliorate hepatic dysfunction among Chinese subjects with diabetes in part through glycemic improvement: a single-center, retrospective, observational study. Diabetes Ther 2018;9:285-295.

91. Kuchay MS, Krishan S, Mishra SK, Farooqui KJ, Singh MK, Wasir JS, et al. Effect of empagliflozin on liver fat in patients with type 2 diabetes and nonalcoholic fatty liver disease: a randomized controlled trial (E-LIFT Trial). Diabetes Care 2018;41:1801-1808.

92. Miyake T, Yoshida S, Furukawa S, Sakai T, Tada F, Senba H, et al. Ipragliflozin ameliorates liver damage in non-alcoholic fatty liver disease. Open Med (Wars) 2018;13:402-409.

93. Ito D, Shimizu S, Inoue K, Saito D, Yanagisawa M, Inukai K, et al. Comparison of ipragliflozin and pioglitazone effects on nonalcoholic fatty liver disease in patients with type 2 diabetes: a randomized, 24-week, open-label, active-controlled trial. Diabetes Care 2017;40:1364-1372.

94. Han E, Lee YH, Lee BW, Kang ES, Cha BS. Ipragliflozin additively ameliorates non-alcoholic fatty liver disease in patients with type 2 diabetes controlled with metformin and pioglitazone: a 24-week randomized controlled trial. J Clin Med 2020;9:259.

95. Nakano S, Katsuno K, Isaji M, Nagasawa T, Buehrer B, Walker S, et al. Remogliflozin etabonate improves fatty liver disease in dietinduced obese male mice. J Clin Exp Hepatol 2015;5:190-198.

96. Qiang S, Nakatsu Y, Seno Y, Fujishiro M, Sakoda H, Kushiyama A, et al. Treatment with the SGLT2 inhibitor luseogliflozin improves nonalcoholic steatohepatitis in a rodent model with diabetes mellitus. Diabetol Metab Syndr 2015;7:104.

97. Shibuya T, Fushimi N, Kawai M, Yoshida Y, Hachiya H, Ito $S$, et al. Luseogliflozin improves liver fat deposition compared to metformin in type 2 diabetes patients with non-alcoholic fatty liver disease: a prospective randomized controlled pilot study. Diabetes Obes Metab 2018;20:438-442.

98. Sumida Y, Murotani K, Saito M, Tamasawa A, Osonoi Y, Yoneda M, et al. Effect of luseogliflozin on hepatic fat content in type 2 diabetes patients with non-alcoholic fatty liver disease: a prospective, single-arm trial (LEAD trial). Hepatol Res 2019;49:64-71.

99. Nozaki Y, Fujita K, Yoneda M, Wada K, Shinohara Y, Takahashi H, et al. Long-term combination therapy of ezetimibe and acarbose 
Kyung-Soo Kim, et al.

Anti-diabetic drugs for NAFLD

for non-alcoholic fatty liver disease. J Hepatol 2009;51:548-556.

100. Komatsu M, Tanaka N, Kimura T, Fujimori N, Sano K, Horiuchi A, et al. Miglitol attenuates non-alcoholic steatohepatitis in diabetic patients. Hepatol Res 2018;48:1092-1098.

101. Ryysy L, Häkkinen AM, Goto T, Vehkavaara S, Westerbacka J, Halavaara J, et al. Hepatic fat content and insulin action on free fatty acids and glucose metabolism rather than insulin absorption are associated with insulin requirements during insulin therapy in type 2 diabetic patients. Diabetes 2000;49:749-758.

102. Mazzotti A, Caletti MT, Marchignoli F, Forlani G, Marchesini G. Which treatment for type 2 diabetes associated with non-alcoholic fatty liver disease? Dig Liver Dis 2017;49:235-240.

103. Belete TM. A recent achievement in the discovery and development of novel targets for the treatment of type-2 diabetes mellitus. J Exp Pharmacol 2020;12:1-15.

104. Sookoian S, Pirola CJ. Nonalcoholic fatty liver disease and metabolic syndrome: shared genetic basis of pathogenesis. Hepatology 2016;64:1417-1420.

105. Sookoian S, Pirola CJ. Review article: shared disease mechanisms between non-alcoholic fatty liver disease and metabolic syndrome - translating knowledge from systems biology to the bedside. Aliment Pharmacol Ther 2019;49:516-527.

106. Sookoian S, Pirola CJ. Genetics of nonalcoholic fatty liver disease: from pathogenesis to therapeutics. Semin Liver Dis 2019;39:124140. 